\title{
BORDERED KLEIN SURFACES WITH MAXIMAL SYMMETRY
}

\author{
BY
}

\author{
NEWCOMB GREENLEAF AND COY L. MAY
}

\begin{abstract}
A compact bordered Klein surface of (algebraic) genus $g \geqslant 2$ is said to have maximal symmetry if its automorphism group is of order $12(g-1)$, the largest possible. In this paper we study the bordered surfaces with maximal symmetry and their automorphism groups, the $M^{*}$-groups. We are concerned with the topological type, rather than just the genus, of these surfaces and its relation to the structure of the associated $M^{*}$-group. We begin by classifying the bordered surfaces with maximal symmetry of low topological genus. We next show that a bordered surface with maximal symmetry is a full covering of another surface with primitive maximal symmetry. A surface has primitive maximal symmetry if its automorphism group is $M^{*}$-simple, that is, if its automorphism group has no proper $M^{*}$-quotient group. Our results yield an approach to the problem of classifying the bordered Klein surfaces with maximal symmetry. Next we obtain several constructions of full covers of a bordered surface. These constructions give numerous infinite families of surfaces with maximal symmetry. We also prove that only two of the $M^{*}$-simple groups are solvable, and we exhibit infinitely many nonsolvable ones. Finally we show that there is a correspondence between bordered Klein surfaces with maximal symmetry and regular triangulations of surfaces.
\end{abstract}

0. Introduction. In the fundamental paper [6] Hurwitz showed that a compact Riemann surface of genus $g \geqslant 2$ has at most $84(g-1)$ automorphisms. Recent research $[7,8,16]$ has studied the values of $g$ for which this bound is attained and the structure of the automorphism group in these cases.

A compact bordered Klein surface [1] of genus $g \geqslant 2$ has at most $12(g-1)$ automorphisms [10]. In this paper we study the surfaces for which this bound is attained, the bordered surfaces which have "maximal symmetry." We also examine the automorphism groups of these surfaces, the $M^{*}$-groups [11]. We are concerned with the topological type, rather than just the genus, of these surfaces and its relation to the structure of the associated $M^{*}$-group.

$\$ 1$ contains preliminary results and definitions, while $\$ 2$ classifies the bordered surfaces with maximal symmetry of low topological genus.

$\S 3$ is the central section of the paper. Surfaces with maximal symmetry are seen to be "full" coverings of those with "primitive" maximal symmetry. The latter surfaces are those whose automorphism groups are " $M^{*}$-simple." The classification problem for surfaces with maximal symmetry then breaks into three parts, which are considered in the next three sections.

Received by the editors February 3, 1981 and, in revised form, September 22, 1981. 1980 Mathematics Subject Classification. Primary 14H30; Secondary 30F10, 57M10.

Key words and phrases. Bordered Klein surface, genus, automorphism, maximal symmetry, $M^{*}$-group, full covering, boundary degree, $M^{*}$-simple group, primitive maximal symmetry, fundamental group, homology group, projective linear group, regular map. 
$\S 4$ discusses techniques for constructing full covers. The manageable constructions are of abelian full covers and their iterates. These constructions yield numerous infinite families of surfaces with maximal symmetry. $\$ 5$ considers the $M^{*}$-simple groups. It is shown that there are only two that are solvable, and infinitely many others are exhibited. However, a full classification is not obtained.

In $\$ 6$ the relation between the topology of a surface with maximal symmetry and the structure of the associated automorphism group is considered. The "index" of the group is seen to correspond to the order of the rotations which are induced on the various components of the boundary. The tool used here is regular maps, and it is shown that bordered surfaces with maximal symmetry correspond biuniquely to regular triangulations.

We should remark that while the surfaces we consider come from analysis or algebraic geometry, this only insures that the automorphisms are reasonably smooth. If $G$ is a finite group of homeomorphisms of a compact bordered surface $X$, then $X$ carries a Klein surface structure that makes $G$ a dianalytic group. While we take the genus $g$ in the sense of algebraic geometry, it is also simply the rank of the fundamental group of the surface.

1. Preliminaries. Klein surfaces are more general than Riemann surfaces in that they need not be orientable and they may have boundary. The basic theory of Klein surfaces is given in [1] where it is shown that compact Klein surfaces correspond to nonsingular algebraic curves over $\mathbf{R}$ just as compact Riemann surfaces do to curves over C. A compact Klein surface $X$ is characterized topologically by orientability, the number $k$ of components of the boundary $\partial X$, and the topological genus $p$. We shall be concerned with compact surfaces with nonempty boundary and for such surfaces the (algebraic) genus $g$ is given by

$$
g= \begin{cases}2 p+k-1, & X \text { orientable } \\ p+k-1, & X \text { nonorientable }\end{cases}
$$

Henceforth by a surface we shall always mean a compact surface. Also, by a bordered surface we shall mean a surface with nonempty boundary.

This paper continues previous investigations $[10,11,12,13]$ into the automorphism groups of bordered Klein surfaces of genus $g \geqslant 2$. The fundamental result about the size of these groups is the following:

TheOREM A [10]. Let $X$ be a bordered Klein surface of genus $g \geqslant 2$ and let $G$ be its automorphism group. Then $G$ is finite and

$$
o(G) \leqslant 12(g-1) .
$$

A bordered surface of genus $g \geqslant 2$ for which the bound (1.2) is attained is said to have maximal symmetry. In [11] there are examples of surfaces with maximal symmetry for infinitely many values of the genus $g$. However, there are an infinite number of values of $g$ for which there is no surface with maximal symmetry [13]. 
Now let $\varphi: X \rightarrow Y$ be a nonconstant morphism of Klein surfaces. For $x \in X$, let $e_{x}$ be the ramification index and $f_{x}$ be the local degree of $\varphi$ at $x$, so that

$$
f_{x}= \begin{cases}2, & \text { if } x \in X^{o} \text { and } \varphi(x) \in \partial Y, \\ 1, & \text { otherwise, }\end{cases}
$$

where as usual $X^{o}=X-\partial X$ denotes the interior of $X$. Then there is an integer $r$ such that $\varphi$ is an $r$-sheeted covering, so that for all $y$ in $Y$

$$
\sum_{\varphi(x)=y} e_{x} f_{x}=r .
$$

Further we have the Hurwitz formula relating the genera $g$ and $\gamma$ of $X$ and $Y$ respectively:

$$
2 g-2=r(2 \gamma-2)+\sum_{e_{x}>1}\left(e_{x}-1\right) n_{x}
$$

where $n_{x}=2$ if $\varphi(x) \in Y^{o}$ and $n_{x}=1$ if $\varphi(x) \in \partial Y$. Thus if $\varphi$ is unramified, then

$$
g-1=r(\gamma-1)
$$

while if $\varphi$ is normal (see $\S 3$ ), then

$$
(2 g-2) / r=2 \gamma-2+\Sigma\left(1-1 / e_{i}\right) n_{i}
$$

where the sum is taken over the finite number of ramified fibers. See [10 and 1].

For any Klein surface $X$, let $A(X)$ denote the automorphism group of $X$. The formula (1.5) was used in [10] to determine the action of the automorphism group of a bordered surface with maximal symmetry.

TheOREM B [10]. Let $X$ have maximal symmetry and let $G=A(X)$. Then the quotient space $X / G$ is the disc $D$ and the quotient map $\pi: X \rightarrow D$ is ramified over four points, all on $\partial D$, with ramification indices $2,2,2,3$.

COROLlARY 1. Let $a_{1}, \ldots, a_{4}$ be the points in $\partial D$ over which $\pi$ is ramified. There are two points, say $a_{1}$ and $a_{2}$, above which the ramification index is two, such that each component of $\partial X$ maps onto the interval $I$ of $\partial D$ from $a_{1}$ to $a_{2}$ which does not contain $a_{3}$ and $a_{4}$. In particular, $G$ acts transitively on the set of components of $\partial X$.

Proof. This is an immediate consequence of the fact that $\pi(\partial X)$ cannot pass through $a_{4}$.

COROLlaRY 2. Let $k$ be the number of components of $\partial X$, let $B$ be a component of $\partial X$, and let

$$
K=\{\sigma \in G \mid \sigma(B)=B\} .
$$

Then $K$ is a dihedral group of order $12(g-1) / k$.

Proof. The action of $K$ on $B$ is easily inferred from the geometry of $\pi: B \rightarrow I$.

Now let $d$ be the number of points of $\pi^{-1}\left(a_{1}\right)$ contained in each component of $\partial X$. If $g$ is the genus and $k$ the number of boundary components of $X$, then (1.3) becomes

$$
12(g-1)=2 d k
$$


Let $p$ be the topological genus of $X$. Then from this equation and (1.1), we obtain the following:

Proposition 1. The number of boundary components $k$ divides $12(p-1)$ when $X$ is orientable and $6(p-2)$ when $X$ is nonorientable.

Except when $X$ is a torus or a Klein bottle with holes, this gives a bound on the possible values for $k$. We shall see in the next section that while there does not exist a Klein bottle with holes with maximal symmetry, there are tori with $k$ holes with maximal symmetry for all $k$ of the form $n^{2}$ or $3 n^{2}$.

A finite group $G$ is called an $M^{*}$-group [11] if it is generated by three distinct elements $t, u, v$ of order two which satisfy the relations

$$
(t u)^{2}=(t v)^{3}=1 \text {. }
$$

It is immediate that 12 divides the order of an $M^{*}$-group $G$. The order of $u v$ is called an index of $G$. A group may have more than one index, corresponding to different sets of generators. The significance of the index will be explored in $\$ 6$. The basic result about $M^{*}$-groups is the following:

THEOREM C [11]. A finite group $G$ is an $M^{*}$-group if and only if it is isomorphic to $A(X)$ for some bordered Klein surface $X$ with maximal symmetry.

The next result says that it is possible to fill in the holes of a bordered Klein surface and obtain a Klein surface without boundary.

THEOREM D. Let $X$ be a bordered Klein surface. Then it is possible to embed $X$ in a Klein surface $X^{*}$ without boundary of the same topological genus, so that every automorphism of $X$ extends to an automorphism of $X^{*}$. The complement of $X$ in $X^{*}$ is a disjoint union of open disks, one for each component of $\partial X$.

Proof. The theorem is a local question and was proved by Oikawa [14,p. 115] when $X$ is orientable and attention is restricted to orientation-preserving automorphisms. Following Oikawa's proof, we reduce to the case of automorphisms of $A=\{z|a \leqslant| z \mid \leqslant b\}$, where $0<a<b$. To obtain the more general result, we need only show that if $\varphi$ is an anti-analytic automorphism of $A$ which takes the interior and exterior boundaries to themselves, then $\varphi$ has a continuation to an anti-analytic automorphism of $\{z|| z \mid \leqslant b\}$. But $\varphi$ must have two fixed points on $\{z|| z \mid=a\}$. Let $z_{0}$ be one of these and let $\rho$ be the reflection of $A$ with axis passing through $z_{0}$. Then $\varphi \rho$ is analytic with a fixed point. Hence $\varphi \rho$ is the identity and $\varphi=\rho$. Then clearly $\varphi$ may be continued to an automorphism of $\{z|| z \mid \leqslant b\}$.

2. Low topological genus. In this section we classify all surfaces $X$ with maximal symmetry for which $X^{*}$ (Theorem D) is a sphere, projective plane, torus, or Klein bottle. This will furnish several examples which illuminate the later theory.

THEOREM 1. Let $X$ be a sphere with $k$ holes with maximal symmetry. Then $k=3,4,6$, or 12 . 
Proof. It follows from Proposition 1 that $k$ divides 12 , and since $X$ is of genus $g \geqslant 2, k \geqslant 3$. Examples of spheres with maximal symmetry with $k=3,4,6,12$ are given in [10 and 11]. The corresponding $M^{*}$-groups, easily determined from the relation of the examples to the platonic solids, are $C_{2} \times S_{3}, S_{4}, C_{2} \times S_{4}$, and $C_{2} \times A_{5}$. Also see [5].

THEOREM 2. Let $X$ be a projective plane with $k$ holes with maximal symmetry. Then $k=3$ or 6 .

Proof. Let $X_{0}$ be the orienting double of $X$ [1, pp. 40-42]. Then $X_{0}$ is a sphere with $2 k$ holes, which is easily seen to have maximal symmetry. Now by Theorem 1 , $k=2,3$, or 6 . If $k=2$, then $X$ is of genus two and $A(X) \cong C_{2} \times S_{3}$, the only $M^{*}$-group of order 12 . But then $A\left(X_{0}\right) \cong S_{4}$, and since $S_{4}$ contains no subgroup isomorphic to $C_{2} \times S_{3}$, the case $k=2$ does not occur. Examples for $k=3,6$ are given in [10 and 11]. The corresponding $M^{*}$-groups are $S_{4}$ and $A_{5}$.

We begin our treatment of tori with holes with an example. Let $\Lambda$ be the lattice subgroup of $\mathbf{C}$ generated by $\{1, \omega\}$, where $\omega=e^{\pi i / 3}$. Let $M=\mathbf{C} / \Lambda$ and let $p$ : $\mathrm{C} \rightarrow M$ be the quotient map. Multiplication by $\omega$ and complex conjugation induce automorphisms of $M$ that generate a group $G \cong C_{2} \times S_{3}$. The point $p(0)$ is a fixed point of $G$. By removing a disc centered at $p(0)$, we obtain a surface $X$ of genus 2 on which $G$ acts, and which thus has maximal symmetry. It is easily seen that this example is unique except for the size of the hole. That is, if $X$ is a torus with one hole with maximal symmetry, then $X^{*} \cong \mathrm{C} / \Lambda$.

THEOREM 3. There is a torus with $k$ holes with maximal symmetry for precisely the following values of $k$ :

$$
k=n^{2} \text { or } 3 n^{2}, \quad n \in \mathbf{N} \text {. }
$$

Proof. Let $X$ be a torus with $k$ holes with maximal symmetry, and let $G=A(X)$. Let $B$ be a component of $\partial X$ and let

$$
H=\{\sigma \in G \mid \sigma(B)=B\} .
$$

Clearly $o(H)=o(G) / k=12$ and $H$ acts on $Z$, where $Z$ is obtained from $X$ by attaching a disc to all boundary components except $B$. By the above remarks $H \cong C_{2} \times S_{3}$ and $Z^{*}=X^{*} \cong \mathrm{C} / \Lambda$, where $\Lambda$ is generated by $\{1, \omega\}$. Let $O$ be the set of centers of the discs adjoined to $X$ to make $X^{*}$, and let $\Omega=p^{-1}(O)$, where $p$ : $\mathrm{C} \rightarrow \mathrm{C} / \Lambda=X^{*}$. It is easy to see that $\Omega$ is a lattice which contains $\Lambda$ as a subgroup of index $k$.

From the action of $H$ on $X^{*}$, it follows that $\Omega$ is invariant under multiplication by $\omega$ and under complex conjugation. Therefore $\Omega$ is similar to $\Lambda$ and is generated by $\{\alpha, \omega \alpha\}$ for some $\alpha$. Comparing the areas of fundamental domains, we see that $|\alpha|^{2} \cdot k=1$. Since $\Lambda \subset \Omega$, there exist integers $m, n$ such that $1=m \alpha+n \omega \alpha$, and then an easy calculation shows that $k=m^{2}+m n+n^{2}$. Since $\bar{\alpha} \in \Omega, \alpha=\omega^{j} \cdot \bar{\alpha}$ for some $j$. Let $\theta=\arg (\alpha)$, and we can assume $0 \leqslant \theta<\pi / 3$. Then $2 \theta=j(\pi / 3)$. Therefore either $\theta=0$ or $\theta=\pi / 6$. Since $\arg (m+n \omega)=-\theta$, either $n=0$ and $k=m^{2}$, or $m=n$ and $k=3 n^{2}$. 
Finally we note that both of these cases can be realized. To obtain $k=n^{2}$, simply set $\Omega=(1 / n) \Lambda$. To obtain $k=3 n^{2}$, let $\alpha=(1+\omega) / 3 n$ as shown below.

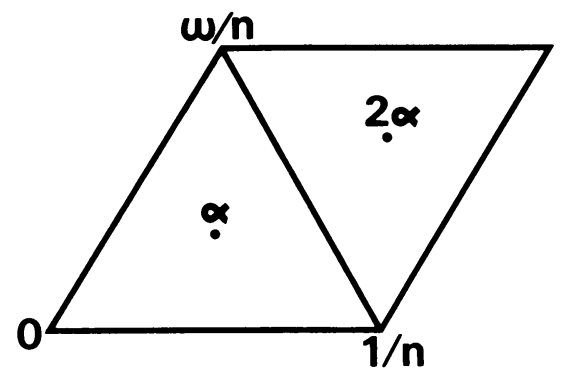

This completes the proof. See also [14].

THEOREM 4. There is no Klein bottle with holes with maximal symmetry.

Proof. Let $X$ be a Klein bottle with $k$ holes. Its orienting double $W$ is a torus with $2 k$ holes. Clearly $W^{*}$ is the orienting double of $X^{*}$, a Klein bottle. Therefore $W^{*}$ is of the form $\mathrm{C} / \Lambda$, where $\Lambda$ is generated by $\{1, \gamma\}$ and $\operatorname{Re}(\gamma)=0$ [1,pp. 61-65]. Therefore $W$ does not have maximal symmetry and so neither does $X$.

COROLlaRY. Let $X$ be a surface with one hole with maximal symmetry. Then $X$ is orientable and of topological genus one, that is, $X$ is a torus with one hole.

Proof. Since $\partial X$ is a circle, $G=A(X)$ is a dihedral group. A simple direct argument shows that the only dihedral $M^{*}$-group is $D_{6} \cong C_{2} \times S_{3}$. Hence $g=2$, and by (1.1) $X$ is either a torus with one hole or a Klein bottle with one hole. The theorem rules out the latter case.

We have exhibited two surfaces of genus two with maximal symmetry: a sphere with three holes and a torus with one. Both have $C_{2} \times S_{3}$ as automorphism group. We now note that this group has two indices. Let $a$ generate $C_{2}$ and let $x, y$ be elements of order two in $S_{3}$. If we set

$$
t=(1, x), \quad u=(a, x), \quad v=(1, y),
$$

then $o(u v)=6$. If we set

$$
t=(1, x), \quad u=(a, 1), \quad v=(1, y),
$$

then $o(u v)=2$. Hence 2 and 6 are indices of $C_{2} \times S_{3}$; see Theorem 17 and its corollary.

3. Maximal symmetry and coverings. Let $\varphi: X \rightarrow X^{\prime}$ be a nonconstant morphism of Klein surfaces. We call $\varphi$ a smooth covering if it is unramified and without folding (so that $X$ is a covering space in the usual topological sense) and a normal covering if the covering transformations act transitively on fibers. If $\varphi$ is smooth and normal and every automorphism of $X^{\prime}$ lifts to an automorphism of $X$, then $\varphi$ is called a full covering. In this case, let $G^{\prime}=A\left(X^{\prime}\right)$ and let $N$ be the group of covering transformations of $X$. Then if we let $G$ be the group of automorphisms of $X$ generated by $N$ and lifts of elements of $G^{\prime}$, we have the exact sequence

$$
1 \rightarrow N \rightarrow G \rightarrow G^{\prime} \rightarrow 1 \text {. }
$$

Of course in general $G$ is only a subgroup of $A(X)$. 
THEOREM 5. Let $\varphi: X \rightarrow X^{\prime}$ be a full covering of the bordered Klein surface $X^{\prime}$. If $X^{\prime}$ has maximal symmetry, then so does $X$. In this case, $G=A(X)$.

Proof. This follows from (3.1), (1.4), and (1.2).

Theorem 5 will be used in $\$ 4$ to produce infinite families of surfaces with maximal symmetry.

THEOREM 6. Let $X$ have maximal symmetry and let $N$ be a normal subgroup of $G=A(X)$ of index $r>6$. Set $X^{\prime}=X / N, G^{\prime}=G / N$, let $\varphi: X \rightarrow X^{\prime}$ be the quotient map, and let $g^{\prime}$ be the genus of $X^{\prime}$. Then

(1) $g^{\prime} \geqslant 2$

(2) $X^{\prime}$ has maximal symmetry;

(3) $G^{\prime} \cong A\left(X^{\prime}\right)$;

(4) $\varphi$ is a full covering.

Proof. To prove (1) we show that $g^{\prime}=0$ and $g^{\prime}=1$ are impossible. From Theorem B and the induced action of $G / N$ on $X / N$ we obtain the following diagram of quotient maps

$$
\begin{aligned}
& X \stackrel{\varphi}{\rightarrow} X^{\prime} \\
& \pi \searrow \quad \downarrow \nu \\
& \text { D }
\end{aligned}
$$

where $\nu$ can be ramified over at most four points of $\partial D$, with ramification indices taken from $\{2,2,2,3\}$. Further, $\nu$ is an $r$-sheeted covering, so that (1.5) for $\nu$ becomes

$$
2 g^{\prime}-2=r\left[-2+\Sigma\left(1-1 / e_{i}\right)\right] \text {. }
$$

It is routine to check that this has no solutions for $g^{\prime}=0$ or $1, r>6$, and $e_{i}$ chosen from $\{2,2,2,3\}$.

Since $G^{\prime} \hookrightarrow A\left(X^{\prime}\right)$, we have $o\left(G^{\prime}\right) \leqslant 12\left(g^{\prime}-1\right)$. Let $g$ denote the genus of $X$. Then applying (1.5) to $\varphi$ yields $g-1 \geqslant o(N) \cdot\left(g^{\prime}-1\right)$, with equality if and only if $\varphi$ is unramified. Thus

$$
o\left(G^{\prime}\right)=\frac{o(G)}{o(N)}=\frac{12(g-1)}{o(N)} \geqslant 12\left(g^{\prime}-1\right) .
$$

It follows that $X^{\prime}$ has maximal symmetry, that $G^{\prime} \cong A\left(X^{\prime}\right)$, and that $\varphi$ is unramified.

To complete the proof we need to show that there is no folding in the covering $\varphi$, i.e., that no interior point of $X$ is mapped to $\partial X^{\prime}$. We use the notation of the corollary to Theorem B. Let $\varphi(x) \in \partial X^{\prime}$. Then $\nu \varphi(x)$ lies in the interval $I$, and this means that $x \in \partial X$. Thus $\varphi$ is a full covering.

An $M^{*}$-group may have quotient groups of order 6 or less, of course, the possibilities being $C_{2}, C_{2} \times C_{2}$, and $S_{3}$. But these are not $M^{*}$-groups, and in these cases the quotient space $X^{\prime}$ is the disc $D$.

Let $\varphi: X \rightarrow X^{\prime}$ be a smooth and normal covering of degree $r$, and let $C_{i}$ be the $i$ th component of $\partial X$. The local boundary degree $d_{i}$ is the number of times $C_{i}$ wraps around its image in $\partial X^{\prime}$. If all of the $d_{i}$ are equal, then the common value $d$ is called the ( global) boundary degree of $\varphi$. In this case, we have

$$
d k=r k^{\prime},
$$


where $k, k^{\prime}$ denote the number of components of $\partial X, \partial X^{\prime}$. In general, of course, a normal covering does not have a global boundary degree. However, if $\varphi$ is a full covering and the surface $X^{\prime}$ has maximal symmetry, note that the boundary degree is well-defined, since then $A(X)$ acts transitively on the components of $\partial X$ and we have (3.1), (3.2).

Theorems 5 and 6 suggest the following definitions. An $M^{*}$-group $G$ is called $M^{*}$-simple if it has no proper $M^{*}$-quotient group, or equivalently, if it has no nontrivial normal subgroup of index greater than six. If $X$ has maximal symmetry and $A(X)$ is $M^{*}$-simple, then we say that $X$ has primitive maximal symmetry. Now Theorem 6 yields

THEOREM 7. If $X$ has maximal symmetry, then it is a full covering of a surface $X^{\prime}$ with primitive maximal symmetry.

In $\$ 4$ we shall give an example which shows that, given $X$, the surface $X^{\prime}$ of Theorem 7 need not be unique.

We can now formulate a program for finding all bordered surfaces with maximal symmetry, which splits naturally into three problems:

1. Find all $M^{*}$-simple groups.

2. Given an $M^{*}$-simple group $G$, find all surfaces $X$ with maximal symmetry (at least up to topological type) for which $G \cong A(X)$.

3. Given a surface $X$ with primitive maximal symmetry, find all full coverings of $X$.

We do not have complete answers for any of these, but we shall return to Problem 1 in $\S 5$, to Problem 2 in $\S 6$, and to Problem 3 in $\S 4$.

A simple $M^{*}$-group is, of course, $M^{*}$-simple. If $G$ is a simple $M^{*}$-group, then $G$ acts only on nonorientable surfaces with maximal symmetry, since otherwise the orientation-preserving maps would be a subgroup of index two in $G$ [10, p. 206]. We shall see in $\$ 5$ that there are infinitely many simple $M^{*}$-groups.

4. Full covers. In this section we use the familiar theory of covering spaces and the fundamental group [9] to construct full covers of a bordered Klein surface. Here we make no assumption about the size of the automorphism group of the surface. However, the immediate applications we have in mind are to surfaces with maximal symmetry.

Let $X$ be a bordered Klein surface of genus $g$ and let $\pi(X)$ denote the fundamental group of $X$. The elements of $G=A(X)$ act on $\pi(X)$, with the action determined only up to inner automorphisms of $\pi(X)$. Then the full covers of $X$ correspond to the normal subgroups of finite index in $\pi(X)$ which are $G$-invariant [9].

All normal $G$-subgroups of finite index in $\pi(X)$ can be obtained by the following general construction. Start with a normal subgroup $N_{0}$ of $\pi(X)$ of finite index and set

$$
N=\bigcap_{\theta \in G} \theta\left(N_{0}\right) .
$$

While this construction does not yield any information on the topological type of the corresponding full cover $Y$ of $X$, it does give the following results. 
Proposition 2. Let $A$ be a finite group which can be generated by $g$ elements, where $g$ denotes the genus of the bordered surface $X$. Then there is a full cover $Y$ of $X$ such that $A$ is a quotient group of the group of covering transformations.

COROLlaRY. Every finite group is a quotient of a normal subgroup of an $M^{*}$-group.

However, we regard a full cover $Y$ of $X$ as determined only when the topological type of $Y$ is found, and we now turn to more manageable constructions. These are obtained by passing to the first homology group $H=H_{1}(X, Z)$, which is a free abelian group of rank $g$. Then the $G$-subgroups of finite index in $H$ correspond to abelian full covers of $X$. Full covers for which the covering group is solvable are produced by iteration.

Let $N$ be a $G$-subgroup of $H$ of finite index $r$, and let $Y$ be the corresponding full cover of $X$. The topological type of the surface $Y$ can be determined as follows. First, from (1.4), the genus of $Y$ is $r(g-1)+1$. Now let $\gamma_{i} \in H$ correspond to a path around the $i$ th component of $\partial X$. The least integer $d_{i}$ such that $\gamma_{i}^{d_{i}} \in N$ is the local boundary degree. If all of the $d_{i}$ have a common value $d$ (as is the case in each of our constructions), then $d$ is the boundary degree of the covering, and $Y$ has $r k / d$ boundary components, where $k$ denotes the number of boundary components of $X$. Finally, $Y$ is nonorientable if and only if there is an element in $N$ corresponding to a path class around which orientation is reversed. Now the topological genus of $Y$ may be calculated from (1.1).

The quotient group $C=H / N$ acts as the group of covering transformations, of course, and $X=Y / C$. If in fact $X$ does have maximal symmetry, then so does the full cover $Y$, and $A(Y)$ is an extension of $A(X)$ by the covering group $C$ :

$$
1 \rightarrow C \rightarrow A(Y) \rightarrow A(X) \rightarrow 1 .
$$

The nonorientable case is somewhat more delicate, and we shall treat the two cases separately.

Let $X$ be orientable of topological genus $p$, and let $\partial X$ have $k$ components. Then $H$ has generators $\alpha_{1}, \beta_{1}, \ldots, \alpha_{p}, \beta_{p}, \gamma_{1}, \ldots, \gamma_{k}$ satisfying the single relation $\gamma_{1} \cdots \gamma_{k}=$ 1. Here $\gamma_{i}$ corresponds to a path around the $i$ th component of $\partial X$, following a given orientation of $X$. Note that if $k=1$, then $\gamma_{1}=1$.

For our first construction we take $N=H^{n}$, the subgroup of $n$th powers of $H$. This gives the following.

THEOREM 8. Let $X$ be orientable of genus $g$. Then for each positive integer $n$ there is a full cover $Y$ of $X$ with

(1) covering group $C \cong C_{n}^{g}$;

(2) boundary degree

$$
d= \begin{cases}n, & \text { if } k>1, \\ 1, & \text { if } k=1\end{cases}
$$

EXAMPLE. Applying this construction to the two surfaces with maximal symmetry of genus two yields two infinite families of surfaces with maximal symmetry. If $X$ is 
a sphere with three holes, then the genus, number of boundary components, and topological genus are

$$
g_{n}=n^{2}+1, \quad k_{n}=3 n, \quad p_{n}=\frac{1}{2} n(n-3)+1 .
$$

If $X$ is a torus with one hole, then

$$
g_{n}=n^{2}+1, \quad k_{n}=n^{2}, \quad p_{n}=1 .
$$

Note that the two families coincide only when $n=3$. The torus with nine holes is a full cover of two surfaces with primitive maximal symmetry.

Note also that when this method is iterated additional examples are obtained. For instance, starting with the sphere with three holes and taking $n=2$, we obtain a sphere with six holes. Applying the construction to this surface, again with $n=2$, yields a surface of genus $2^{7}+1$, which is not of the form $n^{2}+1$. Thus the new surface is outside the first infinite family. This surface is a full cover of the sphere with three holes with a covering group which is solvable but not abelian.

This method improves on that of $[11, \mathrm{pp} .8,9]$ in that it works for all positive integers (not just odd ones) and allows easy determination of topological type. A similar technique was used by Macbeath in [7].

Now let $B$ be the subgroup of $H$ generated by $\gamma_{1}, \ldots, \gamma_{k}$. Then $B$ is a $G$-subgroup of $H$. Indeed for $\theta \in G$, we have $\theta\left(\gamma_{i}\right)=\gamma_{j}^{ \pm 1}$. B is a direct factor of $H$ and is of rank $k-1$, since we have the relation $\gamma_{1} \gamma_{2} \cdots \gamma_{k}=1$.

For our second construction we take $N=H^{n} \cdot B^{m}$, where $m$ divides $n$. From the way that $B$ lies in $H$ we obtain the following.

THEOREM 9. Let $X$ be orientable of genus $g=2 p+k-1$. Then there is a full cover $Y$ of $X$ with

(1) covering group $C \cong C_{n}^{2 p} \times C_{m}^{k-1}$,

(2) boundary degree

$$
d= \begin{cases}m, & \text { if } k>1, \\ 1, & \text { if } k=1,\end{cases}
$$

for any positive integers $m, n$ such that $m$ divides $n$.

Special cases of particular interest are $m=n$, which gives the first construction, and $m=1$, which comes from an unramified cover of $X^{*}$.

For the third construction let $m$ divide $(k, n)$ and set

$$
B(m)=\left\{\gamma_{1}^{e_{1}} \cdots \gamma_{k}^{e_{k}} \mid \Sigma e_{i} \equiv 0(\bmod m)\right\}
$$

It is easily checked that $B(m)$ is $G$-invariant. Then if we take $N=H^{n} \cdot B(m)$ we have the following:

TheOrem 10. Let $X$ be orientable of genus $g=2 p+k-1$. Then there is a full cover $Y$ of $X$ with

(1) covering group $C \cong C_{n}^{2 p} \times C_{m}$,

(2) boundary degree $d=m$

for any positive integers $m, n$ such that $m$ divides $(k, n)$. 
EXAMPLE. If we apply this construction to a sphere with $k$ holes $(k=3,4,6$, or 12 ), we obtain a full cover with boundary degree equal to the degree, and hence $Y$ also has $k$ holes. The following table lists all cases, with $Y$ having genus $g^{\prime}=2 p^{\prime}+$ $k-1$. By results of Sherk [17, pp. 462, 464] and $\S 6$, this table contains all orientable surfaces with maximal symmetry and $k=3,4$, or 6 .

\begin{tabular}{c|c|c|c|c}
$g$ & $k$ & $d$ & $g^{\prime}$ & $p^{\prime}$ \\
\hline 2 & 3 & 3 & 4 & 1 \\
\hline 3 & 4 & 2 & 5 & 1 \\
& & 4 & 9 & 3 \\
\hline & & 2 & 9 & 2 \\
5 & 6 & 3 & 13 & 4 \\
& & 6 & 25 & 10 \\
\hline & & 2 & 21 & 5 \\
& & 3 & 31 & 10 \\
11 & 12 & 4 & 41 & 15 \\
& & 6 & 61 & 25 \\
& & 12 & 121 & 55
\end{tabular}

Now let $X$ be nonorientable. In this case $H$ is generated by $\alpha_{1}, \ldots, \alpha_{p}, \gamma_{1}, \ldots, \gamma_{k}$ with the relation $\alpha_{1}^{2} \cdots \alpha_{p}^{2} \gamma_{1} \cdots \gamma_{k}=1$. Orientation reverses around the path corresponding to $\alpha_{i}$ and is preserved around the path corresponding to $\gamma_{j}$. Let $O$ be the subgroup of $H$ of all elements corresponding to orientation preserving paths. Then

$$
O=\left\{\alpha_{1}^{f_{1}} \cdots \alpha_{p}^{f_{p}} \gamma_{1}^{e_{1}} \cdots \gamma_{k}^{e_{k}} \mid \Sigma f_{i} \equiv 0(\bmod 2)\right\},
$$

$O$ is a $G$-subgroup, and $O$ corresponds to the orienting double cover of $X$. The cover corresponding to a subgroup $N$ of $H$ is orientable if and only if $N \subset O$.

The simplest construction again takes $N=H^{n}$. In this case it yields

THEOREM 11. Let $X$ be nonorientable of genus $g$. Then for each positive integer $n$ there is a full cover $Y$ of $X$ with

(1) covering group $C \cong C_{n}^{g}$,

(2) boundary degree

$$
d= \begin{cases}n, & \text { if } k>1, \\ n /(2, n), & \text { if } k=1 .\end{cases}
$$

Further, $Y$ is orientable if and only if $n$ is even.

EXAMPLE. Let $X$ be a projective plane with three holes, the nonorientable surface with maximal symmetry of genus three. Then for each positive integer $n$ there is a full cover $Y$ of $X$ of genus $2 n^{3}+1$ with $3 n^{2}$ boundary components. If $n$ is odd, then $Y$ is nonorientable of topological genus $2 n^{3}-3 n^{2}+2$. If $n$ is even, then $Y$ is orientable of topological genus $\frac{1}{2}\left(2 n^{3}-3 n^{2}+2\right)$. Again notice how this example improves a result of $[11$, p. 10]. 
Let $B$ be generated by $\gamma_{1}, \ldots, \gamma_{k}$. Then $B$ is of rank $k$ and $H / B \cong C_{\infty}^{p-1} \times C_{2}$. Let $m$ divide $n$ and set $N=H^{n} B^{m}$. Then we have the following:

THEOREM 12. Let $X$ be nonorientable of genus $g=p+k-1$. Then there is a full cover $Y$ of $X$ with

(1) covering group $C \cong C_{n}^{p-1} \times C_{m}^{k-1} \times C_{(2 m, n)}$,

(2) boundary degree

$$
d= \begin{cases}m, & \text { if } k>1, \\ (2 m, n) /(2, n), & \text { if } k=1,\end{cases}
$$

for any positive integers $m, n$ such that $m$ divides $n$. Further $Y$ is orientable if and only if $n$ is even.

The group

$$
B(2)=\left\{\gamma_{1}^{e_{1}} \cdots \gamma_{k}^{e_{k}} \mid \Sigma e_{i} \equiv 0(\bmod 2)\right\}
$$

is a $G$-invariant subgroup of $H$. Let $\delta=\left(\alpha_{1} \cdots \alpha_{p}\right)^{-1}$, so that $\delta^{2}=\gamma_{1} \cdots \gamma_{k}$. It is easily checked that

$$
H / B(2) \cong \begin{cases}C_{\infty}^{p-1} \times C_{4}(\delta), & \text { if } k \text { is odd } \\ C_{\infty}^{p-1} \times C_{2}(\delta) \times C_{2}\left(\gamma_{1}\right), & \text { if } k \text { is even }\end{cases}
$$

Now let $n$ be even and take $N=H^{n} \cdot B(2)$. This gives the following:

TheOREM 13. Let $X$ be nonorientable of genus $g=p+k-1$. Then for each even positive integer $n$ there is a full cover $Y$ of $X$ with

(1) covering group

$$
C \cong \begin{cases}C_{n}^{p-1} \times C_{(4, n)}, & \text { if } k \text { is odd }, \\ C_{n}^{p-1} \times C_{2} \times C_{2}, & \text { if } k \text { is even } .\end{cases}
$$

(2) boundary degree

$$
d= \begin{cases}(4, n) / 2, & \text { if } k \text { is odd } \\ 2, & \text { if } k \text { is even }\end{cases}
$$

Further $Y$ is orientable.

All of the above results can be applied to $M^{*}$-groups to show that if $G$ is an $M^{*}$-group, then certain extensions of $G$ by abelian groups are $M^{*}$-groups. We mention only one case, corresponding to Theorems 8 and 11 .

COROllaRY. If $G$ is an $M^{*}$-group of order $12(g-1)$ and $n$ is a positive integer, then $G$ has an $M^{*}$-extension $G_{n}$ of the form

$$
1 \rightarrow C_{n}^{g} \rightarrow G_{n} \rightarrow G \rightarrow 1 .
$$

These constructions provide us with a wealth of examples of surfaces with maximal symmetry, each presented as an abelian full cover of a surface with maximal symmetry of lower genus. We emphasize, however, that we have not been 
able to classify the abelian full covers of a surface with maximal symmetry. The main result of $\S 6$ is another fruitful source of examples. We shall see that there is a nonorientable surface of genus 11 that is a double cover of the projective plane with 6 holes but that cannot be obtained by the constructions of this section.

5. $M^{*}$-simple groups. Let $\Gamma$ be the extended modular group. $\Gamma$ has generators $T, U, V$ and defining relations $[3, \mathrm{pp} .85,86]$

$$
T^{2}=U^{2}=V^{2}=(T U)^{2}=(T V)^{3}=1 .
$$

It follows that a finite group of order at least twelve is an $M^{*}$-group if and only if it is a homomorphic image of $\Gamma$.

Consider the canonical homomorphism $\varphi_{p}$ from $\Gamma$ to $\operatorname{PGL}(2, p)$, where $p$ is an odd prime. It is easily checked that $\varphi_{p}$ is onto when -1 is a quadratic nonresidue $\bmod p$, and that the image is $\operatorname{PSL}(2, p)$ when -1 is a quadratic residue. Since $\operatorname{PGL}(2,3) \cong S_{4}$ is $M^{*}$-simple, and for $p>3, \operatorname{PSL}(2, p)$ is simple and the only nontrivial normal subgroup of $P G L(2, p)$, we have

THEOREM 14. The following are $M^{*}$-simple groups:

$$
\begin{aligned}
& P G L(2, p), \quad p \equiv 3(\bmod 4), \\
& P S L(2, p), \quad p \equiv 1(\bmod 4) .
\end{aligned}
$$

In $\$ 6$ we shall determine surfaces with maximal symmetry for which these are the automorphism groups. For this purpose we will need to know an index of each group. In $\Gamma$ we may choose

$$
u=\left(\begin{array}{cc}
-1 & 0 \\
0 & 1
\end{array}\right), \quad v=\left(\begin{array}{cc}
-1 & 0 \\
1 & 1
\end{array}\right)
$$

and it is easily computed that $p$ is an index of the image of $\varphi_{p}$.

We know of a few $M^{*}$-simple groups in addition to those in the infinite family given by Theorem 14. First there is, of course, the group $C_{2} \times S_{3}$ of order twelve. Three more $M^{*}$-simple groups appear in the work of Coxeter [2]. He defines $G^{n, p, q}$ as the group with generators $A, B, C$ and defining relations

$$
A^{n}=B^{p}=C^{q}=(A B)^{2}=(B C)^{2}=(C A)^{2}=(A B C)^{2}=1 .
$$

Setting

$$
T=B C, \quad U=C A, \quad V=B C A,
$$

so that

$$
A=T V, \quad B=V U, \quad C=U V T,
$$

we obtain the presentation

$$
T^{2}=U^{2}=V^{2}=(T U)^{2}=(T V)^{n}=(U V)^{p}=(T U V)^{q}=1 .
$$

Hence any finite group $G^{3, p, q}$ is an $M^{*}$-group, and any $M^{*}$-group is a quotient of some group $G^{3, p, q}$ (which need not be finite). From the table of finite groups $G^{n, p, q}$ given in [3, pp. 139, 140], we have the following additional $M^{*}$-simple groups:

$$
G^{3,7,9} \cong \operatorname{PSL}(2,8), \quad G^{3,7,12} \cong \operatorname{PGL}(2,13), \quad G^{3,9,9} \cong \operatorname{PSL}(2,19) .
$$


We have not been able to classify $M^{*}$-simple groups, or even simple $M^{*}$-groups. We do not even know if there exist infinitely many such groups beyond those listed in Theorem 14, although we conjecture that there are. Note that the two smallest $M^{*}$-simple groups, $C_{2} \times S_{3}$ and $S_{4} \cong P G L(2,3)$, are solvable. We now show that they are the only solvable $M^{*}$-simple groups. To do so we shall need the structure of the commutator subgroup of the extended modular group $\Gamma$.

LEMMA 1. The commutator subgroup $\Gamma^{\prime}$ of $\Gamma$ is a free product of two cyclic groups of order three, and $\left[\Gamma: \Gamma^{\prime}\right]=4$.

Proof. Let $\Gamma$ have generators $T, U, V$ with defining relations (5.1), and set $H=\langle T V, T U \cdot T V \cdot T U\rangle$. It is not hard to check that $H$ is normal in $\Gamma$ and that $[\Gamma: H]=4$. The quotient $\Gamma / H$ is abelian, so that $\Gamma^{\prime} \subset H$. But $T V=V T V T$ and $T U \cdot T V \cdot T U=(T U)^{-1} \cdot T V \cdot T U$ are both elements of $\Gamma^{\prime}$. Hence $\Gamma^{\prime}=H$. It is clear that $H$ is the free product of two cyclic groups of order three, since the defining relations for $H$ are $(T V)^{3}=(T U \cdot T V \cdot T U)^{3}=1$.

Corollary. $\left[\Gamma^{\prime}: \Gamma^{\prime \prime}\right]=9$.

Proof. The commutator quotient group $\Gamma^{\prime} / \Gamma^{\prime \prime}$ is the direct product of two cyclic groups of order three [15, p. 249].

Corollary. If $G$ is an $M^{*}$-group, then $\left[G: G^{\prime}\right]$ divides 4 and $\left[G^{\prime}: G^{\prime \prime}\right]$ divides 9 .

LEMMA 2. Let $G$ be a solvable $M^{*}$-group with $\left[G: G^{\prime \prime}\right]=6$, where $G^{\prime \prime}$ is an elementary abelian group. Then $G \cong S_{4}$.

Proof. Since 4 divides $o(G), 2$ divides $o\left(G^{\prime \prime}\right)$. Thus $G^{\prime \prime}$ is an elementary abelian 2-group, and $o(G)$ is of the form $2^{n} \cdot 3$. The quotient $G / G^{\prime \prime} \cong S_{3}$, and it is not hard to see that $G$ has no elements of order larger than 6 . Hence $G$ is a quotient group of a group $G^{3, p, q}$, where $p \leqslant q \leqslant 6$. From the table in [3], we have that $G$ is isomorphic to one of the following groups: $C_{2} \times S_{3}, S_{4}, C_{2} \times S_{4}$. By checking the derived series of these three groups, we see that $G \cong S_{4}$.

THEOREM 15. The only solvable $M^{*}$-simple groups are $C_{2} \times S_{3}$ and $S_{4}$.

Proof. The three smallest (solvable) $M^{*}$-groups, $C_{2} \times S_{3}, S_{4}$, and $S_{3} \times S_{3}$, are the only $M^{*}$-groups of orders $12,24,36$, and $S_{3} \times S_{3}$ is not $M^{*}$-simple. Then let $G$ be a solvable $M^{*}$-group with $o(G) \geqslant 48$. We show that $G$ has an $M^{*}$-quotient group.

Since $G$ is solvable, $\left[G: G^{\prime \prime}\right] \geqslant 6$ by the second corollary to Lemma 1 . Since $o(G) \geqslant 48, G^{\prime \prime} \neq 1$, by the same corollary. If $\left[G: G^{\prime \prime}\right]>6$, then $G / G^{\prime \prime}$ is an $M^{*}$-group. Suppose $\left[G: G^{\prime \prime}\right]=6$. If $G^{\prime \prime}$ were a minimal normal subgroup of $G$, then $G^{\prime \prime}$ would be an elementary abelian group [15, p. 117], contradicting Lemma 2 . Hence $G^{\prime \prime}$ contains a minimal normal subgroup $M$ of $G$ with $[G: M]>\left[G: G^{\prime \prime}\right]=6$, so that $G / M$ is an $M^{*}$-group.

6. Regular maps and maximal symmetry. Let $S$ be a surface without boundary, and let $M$ be a map on $S$, i.e. a polyhedral decomposition of $S$ with $v$ vertices, $e$ edges, and $f$ faces. An automorphism of $M$ is a permutation of its elements which preserves 
incidence. The map $M$ is called regular if its automorphism group $A(M)$ contains

(1) an element $\rho$ which cyclically permutes the edges of some face,

(2) an element $\sigma$ which cyclically permutes the edges meeting at a vertex of this face,

(3) an element $\tau$ which maps some edge to itself and interchanges its vertices while leaving the two bordering faces fixed.

It follows easily from (1) and (2) that $A(M)$ acts transitively on the vertices, edges, and faces of $M$. We say $M$ is of type $\{r, q\}$ if $r$ edges surround a face and $q$ edges meet at a vertex. Clearly

$$
q v=2 e=r f .
$$

The group $A(M)$ maps each face to itself in $2 r$ ways, so that the order of $A(M)$ is $2 r f$. Thus $A(M)$ is generated by $\rho, \sigma, \tau$ and hence also by $\tau, \alpha=\tau \rho$, and $\beta=\alpha \sigma$, which satisfy the relations

$$
\tau^{2}=\alpha^{2}=\beta^{2}=(\tau \alpha)^{r}=(\alpha \beta)^{q}=(\tau \beta)^{2}=1 .
$$

Therefore when $r=3, A(M)$ is an $M^{*}$-group (also when $q=3$, but then we may consider the dual map, of type $\{q, r\})$.

If the surface $S$ has Euler characteristic $\chi<2$, then the regular map $M$ may be derived from another map $N$ of type $\{r, q\}$ on the universal covering space $\tilde{S}$ of $S . N$ is normally called the regular tesselation $\{r, q\}$. The full automorphism group $\Gamma=A(N)$ is generated by three elements $\tau, \alpha$ and $\beta$ with presentation (6.2). Further there is a normal subgroup $K$ in $\Gamma$ such that $\Gamma / K=A(M), N / K=M$, and of course $\tilde{S} / K=S$ (see [3, p. 103] and [20, pp. 765-768]).

We should remark that our definition of regularity is somewhat stronger than the usual one. Most authors, in particular Coxeter and Moser [3], have required only that $A(M)$ contain the rotations $\rho$ and $\sigma$. Maps that also have the symmetry $\tau$ are then called "reflexible". However it is perhaps more natural to require that a regular map possess all of its possible symmetries than just half, and in recent work [20, 21] Wilson gives a definition equivalent to ours. In any case, maps that fail to satisfy (3) are not of interest here. But note that if $S$ is nonorientable, then (1) and (2) actually imply (3).

We now consider the relationship between regular maps and bordered Klein surfaces with maximal symmetry. Let $M$ be a regular map of type $\{r, q\}$ on a surface $S$. The quotient of $S$ under $A(M)$ is a triangle $T$. Let $\pi: S \rightarrow T$ be the quotient map, and let $t$ denote the corner of $T$ whose inverse image is the set of vertices of $M$. Cut off a small (open) corner at $t$ and give the resulting polygonal disc $D$ a Klein surface structure. Then let $X=\pi^{-1}(D) \subset S$. It follows from [1, p. 28] that $X$ has a Klein surface structure and that the restriction of the action of $A(M)$ to $X$ is dianalytic.

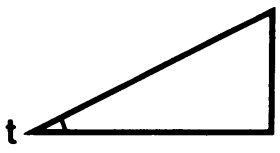

Geometrically the topological type of the bordered surface $X$ is obtained by removing $v$ open discs, each centered about a different vertex of the map $M$. 
Now let $g$ denote the genus of $X$. We need the size of the group $A(M)$ in terms of $g$.

LEMMA 3. $o(A(M))=(g-1) 4 r /(r-2)$, if $r \geqslant 3$.

Proof. By construction, $X$ has $v$ boundary components. If $\chi$ denotes the Euler characteristic of $S$, then an easy calculation shows that $g-1=v-\chi$. But $\chi=v-$ $e+f$. Thus

$$
g-1=e-f=f(r-2) / 2
$$

using (6.1). Hence

$$
o(A(M))=2 r f=(g-1) 4 r /(r-2), \quad \text { if } r \geqslant 3 .
$$

THEOREM 16. Let $M$ be a regular map of type $\{3, q\}$, that is, a regular triangulation. Then the bordered Klein surface $X$ constructed from $M$ has maximal symmetry, and $A(X)=A(M)$.

Note that if a surface $X$ constructed from a regular map of type $\{r, q\}$ has maximal symmetry, then necessarily $r$ divides 6 . If $r=2$, then $g=1$. If $r=6$, then $A(M)$ is a subgroup of index two in $A(X)$.

Theorem 16 provides us with another source of examples of surfaces with maximal symmetry. Set $r=3$, and note that from the proof of Lemma $3, \chi=(6-q) v / 6$. If $q \leqslant 6$, then $\chi \geqslant 0$, and in fact the well-known regular maps of type $\{3, q\}$ on the sphere, the projective plane, and the torus [3, p. 138] can be used to produce the bordered surfaces of $\S 2$. If $q \geqslant 7$, then $\chi<0$ and the universal covering space $\tilde{S}$ of $S$ is the hyperbolic plane $H$. In this case the map $N$ is a $(2,3, q)$ triangulation of $H$ and the group $\Gamma=A(N)$ is an extended triangle group [18, p. 21]. Thus the $M^{*}$-group that acts on the Klein surface $X$ is a quotient of an extended $(2,3, q)$ triangle group.

In all cases it is interesting to note that by varying the size of the removed discs, we actually obtain a one-parameter family of bordered surfaces with maximal symmetry from each regular map of type $\{3, q\}$.

Occasionally we obtain surfaces that cannot be explained by the constructions of $\S 4$. For example, there is a regular map of type $\{3,10\}$ on a nonorientable surface of topological genus six [3,p. 139]. The surface of genus 11 constructed from this map has 6 boundary components and is a double cover of the real projective plane with 6 holes.

Also note that if $M$ is a regular map of type $\{4, q\}$, then the bordered surface $X$ constructed from $M$ has automorphism group of order $8(g-1)$. It follows from [10, p. 207] that if $o(A(X))<12(g-1)$, then $o(A(X)) \leqslant 8(g-1)$. Thus regular maps of type $\{4, q\}$ produce bordered surfaces with automorphism groups of the second largest possible order.

We now start with a bordered Klein surface $X$ that has maximal symmetry and construct an associated regular triangulation. We use Theorem B and its corollaries. Let $g$ and $k$ denote the genus and number of boundary components of $X$, and let $G=A(X)$. Then the quotient space $X / G$ is the disc $D$, the quotient map $\pi: X \rightarrow D$ is ramified above four points $a_{1}, a_{2}, a_{3}, a_{4}$ in $\partial D$ with indices $k_{1}=k_{2}=k_{3}=2$, $k_{4}=3$, and $\pi(\partial X)$ is the interval from $a_{1}$ to $a_{2}$ in $\partial D$. Let $B$ be a component of $\partial X$, 
and let $K=\{\sigma \in G \mid \sigma(B)=B\}$. Then $K$ is a dihedral group, and a generator of the cyclic subgroup of index two acts as a rotation of $B$.

Now fill in the holes of $X$ to obtain a surface $X^{*}$ without boundary so that $G$ acts as a group of automorphisms of $X^{*}$. Let $D^{\prime}$ be the quotient of $X^{*}$ under $G$, and let $\pi^{*}: X^{*} \rightarrow D^{\prime}$ be the quotient map. $D^{\prime}$ is obtained from $D$ by adjoining the quotient of a disc under the dihedral group $K$ :

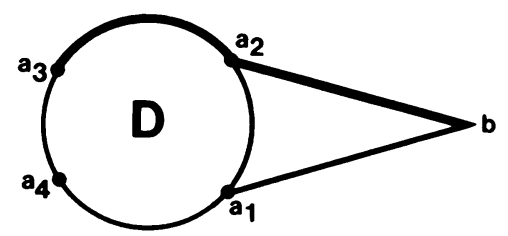

It is easily seen that $\pi^{*}$ is ramified above $a_{3}, a_{4}$, and a third point $b$ in $\partial D^{\prime}$ with indices 2,3 , and $6(g-1) / k$ (the order of the cyclic subgroup of $K$ ).

Let $M$ be the inverse image under $\pi^{*}$ of the interval from $a_{3}$ to $b$ in $\partial D^{\prime}$ which does not contain $a_{4}$. Since the ramification index above $a_{3}$ is $2, M$ is a map on $X^{*}$ with $\pi^{*-1}(b)$ the set of vertices.

THEOREM 17. Let $X$ be a bordered Klein surface with maximal symmetry. Then the associated map $M$ is a regular triangulation of $X^{*}$ with $A(M)=A(X)$.

Proof. Clearly each element of $G=A(X)$ induces an automorphism of $M$ and $M$ is regular. If the map $M$ is of type $\{r, q\}$, then $q=6(g-1) / k$ and we need to show $r=3$. But this follows from $k_{4}=3$, since lifting the triangulation $\left(a_{3}, a_{4}, b\right)$ of $D^{\prime}$ to $X^{*}$ cuts each polygon of $M$ into 6 triangles:

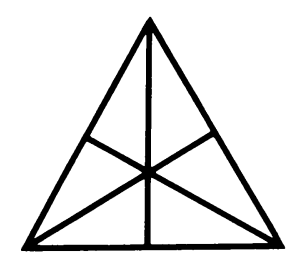

This completes the proof.

Theorem 17 could also be established by using the geometry of the hyperbolic plane $H$ and noneuclidean crystallographic (NEC) groups. Suppose that $X^{*}$ has Euler characteristic $\chi<0$ (the cases of low topological genus were treated in $\S 2$ ). Then we can represent the Klein surface $X^{*}$ in the form $X^{*}=H / K$, where $K$ is a surface group, and further there is an NEC group $\Gamma$ such that $K \triangleleft \Gamma$ and $\Gamma / K \cong G$ (see [11, pp. 2-4; and 4,pp. 23-27]). Now the quotient space $H / \Gamma$ is dianalytically isomorphic to $(H / K) /(\Gamma / K)=X^{*} / G$. The proof of the theorem shows that $X^{*} / G$ is a disc, with the quotient map ramified above three boundary points, the ramification indices being 2,3, and $q$. By applying a useful result of Wilkie [19, p. 96] to the quotient map $H \rightarrow H / \Gamma$, we see that $\Gamma$ is an extended $(2,3, q)$ triangle group. Finally the $(2,3, q)$ triangulation $N$ of $H$ will project to a regular triangulation $M=N / K$ of the surface $X^{*}=H / K$. 
We record the following consequence of the proof of Theorem 17.

Corollary. $q=6(g-1) / k$ is an index of $G=A(X)$.

Proof. This follows from (6.2).

We now see that if an $M^{*}$-group $G$ acts on $X$, then the index $q$ of $G$ is determined by $k$. Further, each component of $\partial X$ is fixed by a dihedral subgroup of $G$ of order $2 q$. Thus the index $q$ of $G$ gives the order of the rotation of each boundary component.

Theorems 16 and 17 establish a bijection between bordered Klein surfaces with maximal symmetry and regular triangulations.

There is also a bijection between regular maps of type $\{4, q\}$ and bordered Klein surfaces of genus $g \geqslant 2$ with $8(g-1)$ automorphisms. This may be established with our techniques and the results in [10, pp. 206, 207].

Finally, we have seen that an $M^{*}$-group $G$ can have more than one index. But the index $q$ determines $k$. Thus if $G$ acts on two topologically different surfaces with the same index, then one must be orientable and the other nonorientable. It would be very interesting to see such an example.

We would like to thank the referee for several helpful suggestions, including the simplified proof of the corollary to Theorem 4 .

\section{REFERENCES}

1. N. L. Alling and N. Greenleaf, Foundations of the theory of Klein surfaces, Lecture Notes in Math., Vol. 219, Springer-Verlag, Berlin-Heidelberg-New York, 1971.

2. H. S. M. Coxeter, The abstract groups $G^{m, n, p}$, Trans. Amer. Math. Soc. 45 (1939), 73-150.

3. H. S. M. Coxeter and W. O. J. Moser, Generators and relations for discrete groups, 3rd ed., Ergebnisse der Mathematik und ihrer Grenzgebiete, Band 14, Springer-Verlag, Berlin-Heidelberg-New York, 1972.

4. W. Hall, Automorphisms and coverings of Klein surfaces, $\mathrm{Ph}$. D. Thesis, University of Southampton, 1977.

5. M. Heins, On the number of 1-1 directly conformal maps which a multiply-connected plane region of finite connectivity $p(>2)$ admits onto itself, Bull. Amer. Math. Soc. 52 (1946), 454-457.

6. A. Hurwitz, Uber algebraische Gebilde mit eindeutigen Transformationen in sich, Math. Ann. 41 (1893), 403-442.

7. A. M. Macbeath, On a theorem of Hurwitz, Proc. Glasgow Math. Assoc. 5 (1961), 90-96.

8. Generators of the linear fractional groups, Number Theory (Houston, 1967), Proc. Sympos. Pure Math., Vol. 12, Amer. Math. Soc., Providence, R.I., 1969, pp. 14-32.

9. W. S. Massey, Algebraic topology: An introduction, Harcourt, Brace \& World, New York, 1967.

10. C. L. May, Automorphisms of compact Klein surfaces with boundary, Pacific J. Math. 59 (1975), $199-210$

11. L Large automorphism groups of compact Klein surfaces with boundary, Glasgow Math. J. 18 (1977), 1-10.

12. A B bound for the number of automorphisms of a compact Klein surface with boundary, Proc. Amer. Math. Soc. 63 (1977), 273-280.

13. Cyclic automorphism groups of compact bordered Klein surfaces, Houston J. Math. 3 (1977), 395-405.

14. K. Oikawa, Notes on conformal mappings of a Riemann surface onto itself, Kodai Math. Sem. Rep. 8 (1956), 23-30; A supplement to "Notes on conformal mappings of a Riemann surface onto itself", ibid. 8 (1956), 115-116.

15. J. Rotman, The theory of groups, Allyn and Bacon, Boston, Mass., 1965.

16. C. H. Sah, Groups related to compact Riemann surfaces, Acta Math. 123 (1969), 13-42.

17. F. A. Sherk, The regular maps on a surface of genus three, Canad. J. Math. 11 (1959), 452-480. 
18. D. Singerman, Symmetries of Riemann surfaces with large automorphism group, Math. Ann. 210 (1974), 17-32.

19. H. C. Wilkie, On non-euclidean crystallographic groups, Math. Z. 91 (1966), 87-102.

20. S. E. Wilson, Riemann surfaces over regular maps, Canad. J. Math. 30 (1978), 763-782.

21 . Operators over regular maps, Pacific J. Math. 81 (1979), 559-568.

Department of Mathematics, Naropa Institute, Boulder, Colorado 80302 (Current address of Newcomb Greenleaf)

DePaRtMent of MAThematics, University of KentuCky, LeXington, KentuCKy 40506

Current address (C. L. May): Department of Mathematics, Towson State University, Towson, Maryland 21204 